\title{
Location Based Services with Location Centric Profiles
}

\author{
M. Ferni Ukrit, B. Venkatesh, Swetabh Suman
}

Department of Software Engineering, SRM University, India

\begin{tabular}{l}
\hline Article Info \\
\hline Article history: \\
Received May 3, 2016 \\
Revised Aug 27, 2016 \\
Accepted Sep 10, 2016 \\
\hline
\end{tabular}

Keyword:

Android

GPS

Location based services

Location centric profiles

\begin{abstract}
In the advancing world of technology, Mobile applications are a rapidly growing segment of the global mobile market. Mobile applications are evolving at a meteor pace to give users a rich and fast user experience. Android operating system supports most applications in today's technical world. It is an open source operating system which highly satisfies the user's needs. In this paper, the applications that are location based i.e. the applications that make use of the Global Positioning System (GPS) is discussed. It is a space based satellite navigation system which provides details of time and location in all weather conditions anywhere on or near earth. We introduce PROFILER, a framework for constructing location centric profiles (LCPs), aggregates built over the profiles of users that have visited discrete locations. In addition to a venue centric approach, we propose a decentralized solution for computing real time LCP snapshots over the profiles of collocated users. In our System we find the corresponding location using Global Positioning and Service Provider also. This is to change the profile mode automatically and alarm notification enable automatically when you are going to reach corresponding location or Regular Location.
\end{abstract}

Copyright () 2016 Institute of Advanced Engineering and Science. All rights reserved.

\section{Corresponding Author:}

M.Ferni Ukrit,

Departement of Software Engineering,

SRM University,

Chennai, Tamil Nadu, India.

Email: fernijegan@gmail.com

\section{INTRODUCTION}

In the pre-smart phone era, cell id's and cell towers were used to find the location of the phone. It has been replaced by the GPS which provides the users with information about their current location and nearby landmarks. GPS is used to search for various places all over the world and nearby places as well [1]. GPS uses triangulation method for calculating user's exact location [2]. The Global Positioning System gives the exact longitude (line parallel to the standard meridian) and latitude (line parallel to the equator) which informs the user about his/her current whereabouts [3].

The GPS concept became a widely deployed and very useful tool for tracking, scientific uses, etc. Global Positioning System is completely based on time. The satellites carry atomic blocks which are very stable and synchronized. GPS satellites transmit the data continuously which provides the current time and position. A GPS receiver coordinates with a number of satellites and equations are solved based on the values received to determine the accurate position of the receiver and its time delay. The localization techniques have recently started to demonstrate significance in performance.

A Location Based Service is an entertainment or information service accessible to mobile devices through the mobile network and utilizing the ability to make use of the geographical position of the mobile device. LBS are used in many contexts such as health, indoor object search, work, entertainment, personal life, etc. LBS can be classified into different categories [4] such as Consumer Services, Emergency Services/public safety, Maps Navigation, Marketing / advertising. LBS are widely utilized concepts. It can be implemented in 
public and safety industry such as emergency service in medical, tracking industry such as fleet management, query the nearest restaurant, navigation with digital maps, payments and so on.

Some of the examples of LBS [4] are:

a. Requesting information about nearest hospitals, petrol pumps, etc.

b. Requesting information about ATM's or restaurants nearby.

c. Traffic related information.

Recently, mobile application named Bus Route Planning was developed to help the user to get information about public transportation using bus [5]. In [6] the android base application of Augmented Reality that has feature to support the action of a bus user in an innovative and dynamic ways by putting additional information layer on smart phone camera screen that gives the instruction assistant that leading the user way to the nearest bus stop was described.

The existing system is to find the location only using GPS and the service provider [7]. The GPS sends to the mobile device a combination of latitude and longitude like $\mathrm{X}, \mathrm{Y}$ axis which informs the user about his/her current location [2]. This system only finds the corresponding location using GPS. It fails to make any changes in any other application installed in the smart phone. It does not change or update any application depending on the location of the mobile device, such as alarm notification, changing profile, managing sound automatically, etc. Thus, the full potential of the Global Positioning System remains unfulfilled. The traditional methods were:

a. GSM Localization: GSM localization means finding the location of a mobile device in relation to its cell site. It involves multi-iteration of the signal from cell towers serving the mobile device.

b. Near LBS: This method uses the local range technologies such as Bluetooth, WLAN, infrared, etc to match devices to nearby services.

c. Control Plane Locating: Service providers get the location of the mobile device based on the radio signal delay of the closest cell phone towers (for phones without GPS).

The system only finds the corresponding location using GPS (combination of latitude and longitude). This is completely a manual system where there is no notification after reaching particular location. Profile is changed based on time. Geographic routing protocols use location information when they need to route packets. In [8] routing protocols is combined with location-based services in order to reduce communication establishment latency and routing overhead. Privacy preservation has recently received considerable attention for location-based mobile services. Various location cloaking approaches have been proposed to protect the location privacy of mobile users [9]. In [10] a driving direction for finding optimal route based on the GPS is presented. In this system, GPS equipped taxis are employed as devices send location information to a centralized sever. The real-time data are used to monitor and mitigate natural hazards arising from earthquakes, volcanic eruptions, landslides, and coastal sea-level hazards in the Pacific Northwest [11]. In future a system can be implemented for municipal waste with the help of GIS [12].

In the proposed system user can manage the profiler and alert automatically based on the location centric profiles (LCPs) value that means latitude and longitude. In our project we can maintain the one database which contain some of the location centric profiles (LCPs) and that matching ideals, we can find the distance between the current location and corresponding instance location, whether that satisfy our condition which will automatically convert prefer mode (silent, vibrate or normal) of the profile of the smart phone and alert also.

This paper is organized as follows: Section II explains the methodology used and Section III explains the results and discussion for the proposed methodology.

\section{METHODOLOGY USED}

\subsection{Overview}

Location services provide the user location based services such as profiling. Profile of mobiles like configuration parameters for many things and examples of profiles are silent, outdoor etc. People needs to manage these profiles as per their schedule but needs to be done manually and always needs an update after the action is over, thus an application is proposed to solve this problem. In the proposed system user manages profile automatically. This is based on the Location Centric Profiles (LCPs) i.e. on latitude and longitude values. A database is maintained which contains some of the LCPs and corresponding locations. The user creates a profile for each location and can also create many profiles. In the proposed system user needs to select a location in which the profile, ringtones and wallpapers that has to be changed. Once when the saved location is reached, a background task will be running to calculate the distance between current and saved locations as shown in Figure 1. When the certain range is reached, the profile will be automatically changed according to user.

IJECE Vol. 6, No. 6, December 2016 : $3001-3005$ 


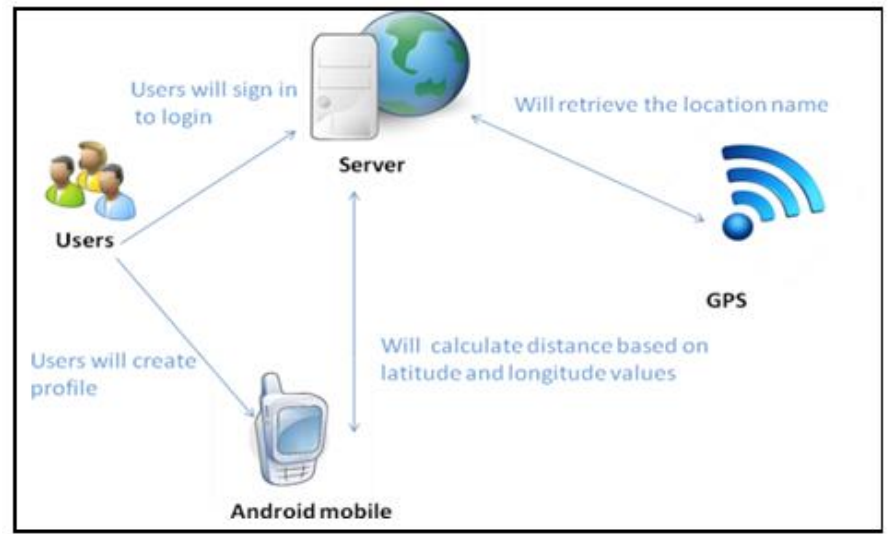

Figure 1. System Architecture

\subsection{Create New Profile}

This module is mainly used to create a profile. The profile which contains profile name, wallpaper, and ringtone and destination location. The user can create any number of profiles. At first the user should provide a name for his profile, the user can have same name for more than one profile. Now the user should select a ringtone for their profile. Ringtone can be selected both from phone memory and also from SD card.Then the wallpaper has to be chosen. It has an option of getting wallpaper from default memory and from SD card. Now we have to choose the location. Through GPS the current location is to be displayed. From the map, the user can choose the destination location by moving the pin icon. The destination location is to be displayed. Then the user needs to save the profile. A message New Profile has been created will be displayed. Then the created profile is displayed in the list.

\subsection{Get GPS}

In this module through GPS the location of the address will be displayed. When the location is chosen by the user, it automatically shows the current location of the user. The user needs to long press the pin icon which shows the current location. Now the icon to be dragged and the destination location have to be chosen. When the user places the icon in the destination place and enters ok the location address will be displayed in the profile creation process. A background task is been running to calculate the current location distance and saved location distance. Thus the location is saved.

\subsection{Location Service}

This module shows the users current location. There is an option of getting the latitude and longitude from maps. The user can choose whether profile has to be changed. The current location is displayed in the profile. Then profile is to be saved.

\subsection{Profile DataBase}

In this module, the users profile is being saved in the database. By clicking the profile setting we can sort the profile according to the users query. Database has the all information of the profiles saved. The GPS location, ringtone, wallpaper is stored as a profile in database. The profile is saved in the database.

\subsection{Profile Management}

In the module the user is allowed to manage the database. The user also can change the settings of the profile. The user can add a profile or delete a profile. The managing of the profile takes long so the option of managing the profile is introduced. This is the use of profile management.

\section{RESULTS AND DISCUSSION}

The proposed methodology has been implemented in Eclipse 3.4. This application can be used in Android 5.1 lollipop. The implementation results have been shown in Figure 2 and Figure 3. 


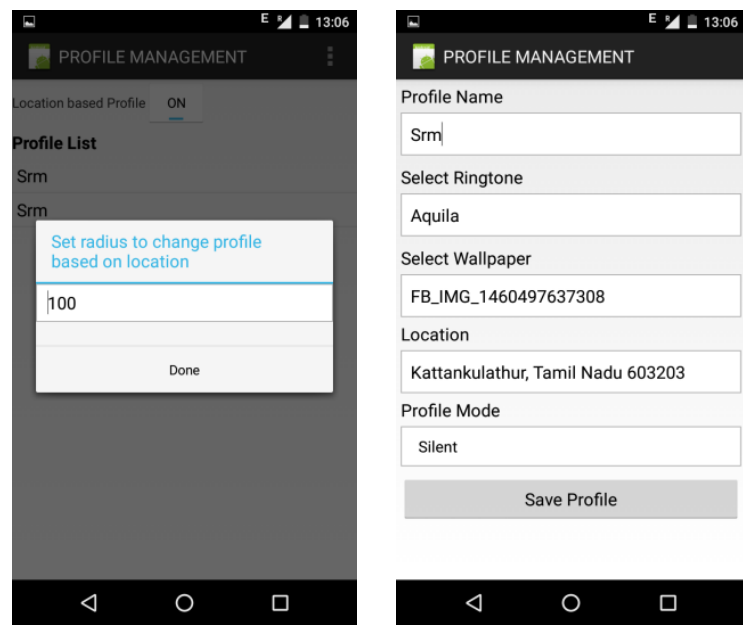

Figure 2. Profile Management

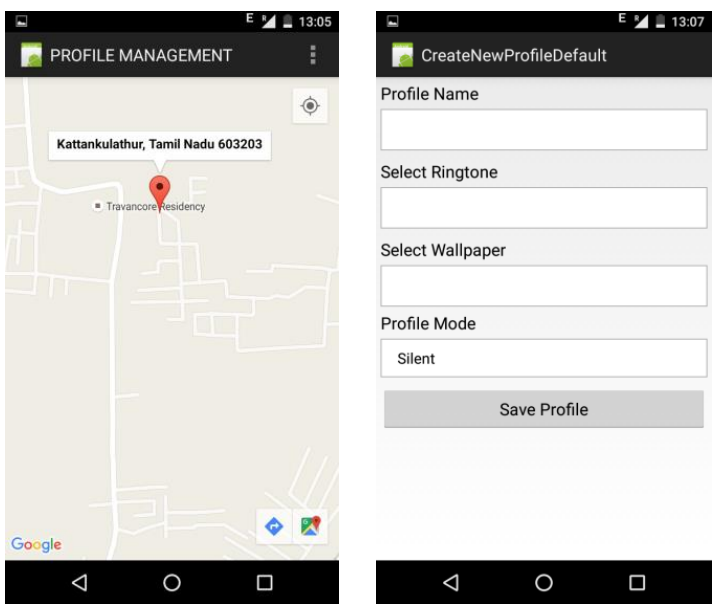

Figure 3. Create New Profile

The user can choose the profile name, ringtone, wallpaper and the desired location. We can also set the profile mode. The location is found by using GPS and Service Provider. This is an automatic system where the profile mode is changed with alarm notification. Volume and wallpaper are also changed. Many numbers of profiles can be created. Many numbers of locations can be saved. Location is fetched accurately.

In today's improvising world, people tend to reduce their work effort and also save time. This application intelligently and accurately provides services as per locations reducing human efforts. It maintains location information within its neighborhood region defined as Neighborhood Update (NU), updating its location information to the distributed location server network which is also called Location Server Update (LSU).

In this way a systematic way of profile changing according to GPS coordinates occurs automatically making the user more convenient. Hence the automatic switching of mobile profile offers a unique way of tracking locations and managing different user profiles according to need.

This application can be used in various places like Hospitals, Petrol pumps, Universities, Corporate offices etc. to automatically switch over to the mode which the user has set for it.

\section{FUTURE WORK}

The proposed system can be further enhanced by introducing automatic messaging feature which can be used to send message automatically to a predefined recipient when certain location is reached and the same can be done through alerts. A group can also be created consisting of users of a particular location so 
that whenever a user of that group reaches a predefined location set for that group, it would automatically manage user profiles.

\section{REFERENCES}

[1] H. Hassanieh, et al., "Faster GPS via the sparse Fourier transform," Proceedings of ACM MobiCom., pp. 353-364, 2012.

[2] S. Mascetti, et al., "Privacy in geo-social networks; Proximity notification with untrusted service providers and curious bundles," VLDB.j, vol/issue: 20(4), pp. 541-566, 2011.

[3] M. M. Hoque and N. Jakowski, "An alternative ionospheric correction model for global navigation satellite systems," Journal of Geodesy, Springer, 2014.

[4] A. Kushwaha and V. Kushwaha, "Location Based Services using Android Mobile Operating System," International Journal of Advances in Engineering \& Technology, vol/issue: 1(1), pp. 14-20, 2011.

[5] A. H. Brata, et al., "Software Development of Automatic Data Collector for Bus Route Planning System," International Journal of Electrical and Computer Engineering, vol/issue: 5(1), pp. 150-157, 2015.

[6] K. C. Brata, et al., "Location-Based Augmented Reality Information for Bus Route Planning System," International Journal of Electrical and Computer Engineering, vol/issue: 5(1), pp. 142-149, 2015.

[7] H. Gao and H. Liu, "Data Analysis on location based Social Networks," Mobile social networking, Springer, 2014.

[8] M. Ayaida, et al., "Joint routing and location-based service in VANET'S," Journal of parallel and distributed computing, vol/issue: 74(2), pp. 2077-2087, 2014.

[9] X. Pan, et al., "Distortion -based anonymity for continuous queries in location based mobile services," in Proc. GIS, pp. 256-265, 2009.

[10] A. Renugambal and V. A. Kameshwari, "Finding optimal vehicular Route Based on GPS," International Journal of Computer Science of Information Technologies, vol/issue: 5(2), pp. 1330-1332, 2014.

[11] R. Popovici, et al., "Real time monitoring of techtonic displacements in the Pacific Northwest through an array of GPS receivers," International Journal of Computers Communications \& Control, vol/issue: 10(1), pp. 78-88, 2015.

[12] V. E. N. Mariappan and P. Selvi, "GIS based municipal solid waste management solution for Kanchipuram Muncipality," Journal of Advanced Research in Civil and Environmental Engineering, vol/issue: 2(3), pp. 17-22, 2015 . 